Published in final edited form as:

Eur Urol. 2013 June ; 63(6): 1128-1129. doi:10.1016/j.eururo.2013.03.017.

\title{
Dysregulation of cholesterol homeostasis in human prostate cancer through loss of ABCA1:
}

Cholesterol and Aggressive Prostate Cancer: No Way Out

Keith R. Solomon, Emma H. Allott, Michael R. Freeman, and Stephen J. Freedland

\section{Experts' summary}

In this manuscript Lee at al. explore the regulation and loss of ABCA1, a transmembrane transporter that serves as a key mediator of cellular cholesterol efflux. Towards this end, the authors demonstrate that the promoter of the $A B C A l$ gene is epigenetically silenced by hypermethylation in the human prostate tumor cell line, $\mathrm{LNCaP}$, but not in a second prostate tumor cell line, DU145. Indeed, they demonstrate that LNCaP cells have higher basal cholesterol levels relative to DU145 cells. Demethylation (using 5-aza-2'-deoxycytidine) and transactivation of the $A B C A 1$ promoter (using T0901317, a liver $\mathrm{X}$ receptor agonist) restores $A B C A 1$ gene and protein expression in $\mathrm{LNCaP}$, resulting in increased cholesterol efflux and reduced intracellular cholesterol levels. Together, these results suggest that loss of ABCA1 expression leads to a dramatic alteration in cholesterol homeostasis. The authors go on to reveal that this is not merely a cell culture phenomenon; $A B C A 1$ promoter hypermethylation accompanied by reduced expression of this key transporter protein is associated with more aggressive human prostate cancer (as measured by Gleason grade).

\section{Experts' comments}

Cholesterol is necessary for many functions in mammalian cells. Consequently, under normal conditions intracellular cholesterol content is carefully regulated through processes of synthesis, uptake and efflux, with efflux carried out mainly by ABCA1 and ABCG1 (ATP-binding cassette transporters). Despite this regulatory machinery, dysregulation in the cholesterol content of prostate cancers relative to normal tissue was initially described by Swyer some seventy years ago [1]. Since then, numerous analyses of human subjects and experimentation using pre-clinical models have supported the existence of a relationship between cholesterol and prostate malignancy, with recent evidence suggesting that higher levels of circulating cholesterol are positively associated, while the use of cholesterollowering statin drugs is inversely associated with developing aggressive prostate cancer [2]. The novelty of Lee et al. is in the description of the mechanism that explains the phenomenon of increased intraprostatic cholesterol. Moreover, the observations of Lee et al. may provide the fundamental link between results of studies reporting on high circulating cholesterol levels and tumor growth [2] and those describing high levels of intraprostatic cholesterol in the malignant prostate [3]. Indeed, prior analyses have shown that experimental hypercholesterolemia leads to cholesterol accumulation in both the normal prostate and in prostatic tumors $[4,5]$. 
Increases in the cholesterol content of prostate cancers may contribute to tumor progression by altering the membrane dynamics of malignant cells, inducing inflammatory responses, promoting cell proliferation, providing substrate for cell membrane biosynthesis, and potentiating the synthesis of androgens. While such processes promote tumor growth in all stages of disease, they may be of vital importance in promoting the most aggressive cancers [2].

Prior studies have noted that $\mathrm{LNCaP}$ cells express a low level of ABCA1 [6-8]. The current study of Lee et al. demonstrates a compelling mechanism behind the loss of ABCA1 expression that may explain in part the accumulation of cholesterol in the malignant prostate.

By providing a partial mechanism to explain cholesterol accumulation in malignant prostate cells and by demonstrating the relevancy of their finding to human tumors, the authors not only have buttressed the reliability of the observations made concerning cholesterol and prostate cancer, but may have also provided new therapeutic targets. Could cholesterol accumulation in the prostate be reversed by targeted demethylation? Could gene therapy using an unmethylated $A B C A 1$ transporter construct be attempted? Could other methods of cholesterol reduction, such as the use of statin drugs or ezetimibe [5], be accomplished to offset the loss of ABCA1? Regardless of the manner of therapy, this important study reinforces the idea that cholesterol, its synthetic precursors, synthetic enzymes and transport apparatus may be targets for combating prostate cancer.

In summary, the current study suggests that epigenetic silencing of the $A B C A 1$ gene in prostate cancers may contribute to the accumulation of cholesterol commonly observed in aggressive prostate tumors, a phenomenon believed to stimulate their growth. This suggests that the malignant prostate may be particularly sensitive to excess cholesterol because once in, cholesterol has a hard time finding its way out.

\section{References}

1. Swyer G. The cholesterol content of normal and enlarged prostates. Cancer Res. 1942; 2:372-5.

2. Pelton K, Freeman MR, Solomon KR. Cholesterol and prostate cancer. Curr Opin Pharmacol. 2012

3. Schaffner CP. Prostatic cholesterol metabolism: regulation and alteration. Prog Clin Biol Res. 1981; 75A:279-324. [PubMed: 6175978]

4. Kim J, Di Vizio D, Kim TK, et al. The response of the prostate to circulating cholesterol: activating transcription factor 3 (ATF3) as a prominent node in a cholesterol-sensing network. PloS One. 2012; 7:e39448. [PubMed: 22768301]

5. Solomon KR, Pelton K, Boucher K, et al. Ezetimibe is an inhibitor of tumor angiogenesis. Am J Pathol. 2009; 174:1017-26. [PubMed: 19179610]

6. Pommier AJ, Alves G, Viennois E, et al. Liver X Receptor activation downregulates AKT survival signaling in lipid rafts and induces apoptosis of prostate cancer cells. Oncogene. 2010; 29:2712-23. [PubMed: 20190811]

7. Trasino SE, Kim YS, Wang TT. Ligand, receptor, and cell type-dependent regulation of ABCA1 and ABCG1 mRNA in prostate cancer epithelial cells. Mol Cancer Ther. 2009; 8:1934-45. [PubMed: 19531574]

8. Leon CG, Locke JA, Adomat HH, et al. Alterations in cholesterol regulation contribute to the production of intratumoral androgens during progression to castration-resistant prostate cancer in a mouse xenograft model. Prostate. 2010; 70:390-400. [PubMed: 19866465] 